\title{
Molecular Pathways of Colorectal Carcinogenesis are Promising Mistery?
}

\author{
Renata Dobrila Dintinjana*1 ${ }^{* 1}$ Arnela Redzović ${ }^{2}$ and Marijan Dintinjana ${ }^{1}$ \\ ${ }^{1}$ Department for Radiotherapy and Oncology, Clinical Hospital Centre Rijeka, Croatia \\ ${ }^{2}$ Department of General Practice, Clinical Hospital Centre Rijeka, Croatia
}

*Corresponding author: Renata Dobrila Dintinjana, Department for Radiotherapy and Oncology, Clinical Hospital Center Rijeka,Krešimirova 42, 51000 Rijeka, Croatia, Tel: 0038598257 729; Fax: 0038551658 391; E-mail: renatadobrila@windowslive.com

Recieved date: December 2, 2013, Accepted date: April 17, 2014, Published date: April 25, 2014

Copyright: (c) 2014 Dintinjana RD, et al. This is an open-access article distributed under the terms of the Creative Commons Attribution License, which permits unrestricted use, distribution, and reproduction in any medium, provided the original author and source are credited.

\begin{abstract}
The specific etiologic factors and pathogenetic mechanisms, underlying the development of cancers of the colon and rectum appear to be complex and heterogeneous. Despite the continuous advancement in diagnostic and therapeutic methods, despite the global and national programs for prevention it is evidenced increase in colorectal cancer (CRC) incidence and mortality.Further progress is needed in the field of non-invasive diagnostic methods to enable early diagnosis, pre and postoperative staging, and to assist in selecting the most suitable neo-adjuvant and adjuvant therapeutic methods and post-treatment follow-up. There are attempts to "personalise and individualise" anticancer therapy based on presence or absence of specific biomarkers. In this review we will focus on the prevalence, the latest progress made within the genomic and proteomic fields and their significance as possible prognostic and predictive markers in CRC patients.
\end{abstract}

Keywords: Colorectal cancer; Carcinogenesis; Tumour suppressor genes; Genomic instability

\section{Introduction}

Colorectal cancers (CRC) are the third most common human malignancy, and are also the leading cause of cancer-related deaths worldwide [1]. Recent advances in detection, chemotherapeutic and biological agent based therapies, combined with liver resection have dramatically increased the survival rate of CRC patients [1]. However, CRC still remains an uncontrollable disease [2]. Overall, the lifetime risk of developing CRC is about 1 in 20 ( 1 in 17 for men and 1 in 26 for women $(5.1 \%)$, and the mortality rate from CRC is also alarming. Relative CRC risk is defined by genetic predisposition and environmental factors, with age being the most important risk factor for sporadic CRC ( $>90 \%$ of sporadic CRCs occurs in individuals over the age of 50). Other risk factors include family history of CRC, a diet low in fibres and folate and high in fat and red meat, alcohol, cigarette smoking, sedentary occupation, obesity and diabetes. Approximately $5 \%$ of all CRC are due to inherited genetic mutations. Of the remaining $95 \%$ of cases, about $20 \%$ have a positive family history but cannot be categorized to any hereditary CRC syndrome. These are probably caused by genetic alterations secondary to an inherited predisposition, or common dietary and environmental factors [1-3].

\section{The Adenoma-Carcinoma Sequence}

CRCs evolve through a stepwise accumulation of genetic and epigenetic alterations, leading to the transformation of normal colonic mucosa into invasive cancer. Most CRCs arise within pre-existing adenomas which harbour some of the genetic fingerprints of malignant lesions [4]. This transformation is continuing during 10-15 years.The specific factors and pathogenetic mechanisms underlying the development of CRCs are complex and heterogeneous. Etiologic factors include environmental and dietary exposures; still the major challenge is defining specific agents that influence risk of CRCs [5].
Some encouraging advances are visible:specific gene defects that underlie several inherited forms of CRCs are defined, new insights into the constellation in molecular alterations for initiation and progression of sporadic tumours have been done, and the prevalence and mutations in several oncogenes and tumour suppressor genes are discovered.

The adenomatous polyp or adenoma is important precursor lesion to cancer [6]. Only a fraction of adenomas progress to cancer and adenomas $>1 \mathrm{~cm}$ in size are estimated to have a $15 \%$ chance of progression to carcinoma during a 10 year period [7].

Individuals affected by syndromes (i.e. familial adenomatous polyposis) that strongly predispose to the development of adenomas, develop CRCs by the third to fifth decades of life.

\section{Sporadic Colorectal Cancer}

Genetic model of colorectal tumorigenesis is based on the oncogene and tumour suppressor gene alterations. Development of CRC is accordance of series of events leading to the transformation of normal mucosa to adenoma and then to carcinoma. Genomic instability is an integral part in this transformation process. Three distinct molecular pathways are important: Chromosomal Instability (CIN) pathway, Microsatellite Instability (MSI) pathway, and the CpG Island Methylator Phenotype (CIMP) pathway [8,9]. Some tumours are exhibiting features of more than one pathway.

K-ras, B-Raf and PI3Kca remains the only specific alterations detected in a high percentage of CRCs of any stage [10]. Focal gains or losses are found in regions containing important cancer genes, e.g. VEGF, MYC, MET, LYN, PTEN, and others [11]. Most amplifications have been identified on chromosomes $7,8 \mathrm{q}, 13 \mathrm{q}, 20$, and $\mathrm{X}$, and most deletions on chromosomes $1,4,5,8 \mathrm{p}, 14 \mathrm{q}, 15 \mathrm{q}, 17 \mathrm{p}, 18,20 \mathrm{p}$, and 22q. Chromosomes 1, 5, 8, 17, and 18 have the highest frequency of allele loss $(46-78 \%)$ and whole chromosome loss is more frequent for chromosome 18 [12-13]. 


\section{Chromosomal instability}

The most common cause of genomic instability in CRC is chromosomal instability (65-70\% of sporadic CRCs). Gain or loss of whole chromosomes or chromosomal regions harbouring genes integral for the process of CRC carcinogenesis are specific for CIN which is coming out from defects in chromosome segregation with subsequent aneuploidy, telomere dysfunction, and/or defects in the DNA damage response mechanisms[14]. Higher levels of CIN are constantly associated with poorer disease free survival (DFS) [15].

\section{Microsatellite instability}

$20 \%$ mismatch repair function is activated either by somatic mutations or epigenetic inactivation leading to microsatellite instability (MSI) [16,17]. Microsatellites are short repeat nucleotide sequences that are spread out over the whole genome [16,17]. Because of their repetitive manner they are prone to errors during replication. These errors are recognised and repaired by the DNA mismatch repair (MMR) system $[18,19]$. MSI is a consequence of the inability of the MMR system to correct errors and is reflected by frame shift mutations in the microsatellite repeats. Members of the MMR system which have been identified are MSH2, MLH1, MSH6, PMS2, MLH3, MSH3, PMS1, and Exo1.73. In about $60 \%$ of all CRCs, another form of MSI (EMAST-elevated microsatellite alterations at selected tetranucleotide repeats) is present [20].

There have been fewer mutations in K-ras and p53 [21]. In sporadic MSI-high CRCs, B-Raf V600E mutations are often founded [21]. Another one frequent mutation (in $90 \%$ of CRC with MSI) can be found; it is mutation in the polyadenine tract of transforming growth factor $b$ type II receptor (TGFbRII) which inactivates gene function.

In the presence of such defective MMR function many genes are susceptible to mutations (also genes which are containing coding repeats involved in DNA signal cell cycle, and the transcription factors ) [21].

In clinical setting, an improved survival has been reported with adjuvant fluorouracil chemotherapy in MSI CRCs of germline origin but not in sporadic cases.Less data are available on predictive value of MIS on response to oxaliplatin based adjuvant chemotherapy [22].

\section{K-ras}

$30-60 \%$ of CRC has mutated K-ras proto-oncogene with mitogenactivated protein kinase (MAPK) signalling activated as a consequence of mutation in KRAS gene $[23,24]$. Mutations in KRAS, frequently in codon 12 and 13 and less common in codon 61, 63 and 146 are major predictive biomarkers for resistance to anti- EGFR treatment [23-25].Activated K-ras (through activation of downstream targets including BCL-2, H2AFZ, RAP1B, TBX19, E2F4, and MMP1) also play a role in the transition from adenoma to carcinoma [24].

PIK3CA mutation is found in $14 \%$ of CRCs. These tumours are mostly found at proximal colon, they are frequently mucinous, and associated with K-Ras mutation. CRCs with K-Ras mutation have mucinous differentiation more frequently than CRCs with wild-type K-Ras [26].

\section{Tumour suppressor gene defects}

Loss of heterozygosis $(\mathrm{LOH})$ is a principal mechanism for one allele inactivation of certain tumour suppressor gene in cancer. Most CRCs
(75\%) have allelic loss of chromosome 17p [27]. The $17 \mathrm{pLOH}$ is targeting p53 gene for inactivation. The mutation of $\mathrm{LOH}$ of p53 is arising most frequently during the transition of adenoma/carcinoma sequence and may facilitate continued growth and the acquisition of invasive properties in CRCs [28]. Loss of $17 \mathrm{p}$ is a late event in the process of colorectal tumorigenesis, not reported in adenomas but in $75 \%$ of CRCs [29]. The p53 is a transcription factor with tumour suppressor activity that binds to a specific DNA sequence and activates a number of genes involved in cell cycle arrest, apoptosis, senescence, autophagy, and cellular metabolism. The p53 facilitates the cellular adaptation in response to different cellular stresses including DNA damage by mutagens, oncogenic stimulation, hypoxia, and telomere erosion [30].

$20-50 \%$ of sporadic CRC has allelic loss of chromosome 5q [31]. APC and the Mutated in Colorectal Cancer (MCC) genes are located on the long arm of chromosome 5. Somatic APC mutations are found in $60-80 \%$ of CRCs as well as in a large percentage of adenomas, indicating that APC mutation is an early event in the process of colorectal tumorigenesis [32]. APC belongs to the canonical Wnt/ wingless pathway and APC protein forms a complex with b-catenin, axin, and glycogen synthase kinase 3 (GSK3) [33]. Activation of the Wnt signalling pathway is a common feature in cancers and leads to its development, progression and metastasis. Wnt $/ \beta$-catenin signalling pathway is interconnected with the MicroRNA-mediated gene regulation, forming a $\mathrm{Wnt} / \beta$-catenin-microRNA regulatory network, and this is important for oncotherapy which is targeting the $\mathrm{Wnt} / \beta$ catenin pathway [34,35]. Although it is known that activation of $\beta$ catenin and PI3K pathways are crucial points in the oncogenesis of CRCs, it still remains unknown whether these two pathways functions independently or cooperatively [36]. No association between polymorphisms in regions of frequent mitotic recombination on $5 \mathrm{q}$ and CRC risk was found, suggesting that local influences over the rate of loss of heterozygosis at APC can't explain inter-individual differences in susceptibility to colorectal cancer [37].

About $50-70 \%$ of primary CRCs and nearly $100 \%$ liver metastases express LOH of chromosome 18q. Loss of this locus increases the potential for metastasis and therefore this is a marker of poor prognosis in stages II/III of CRCs. Inactivation of a chromosome 18q tumour suppressor gene has a role in the later stages of carcinogenesis, cancer progression and metastasis [38-40]. SMAD4 and SMAD2 genes are tumour suppressor genes on chromosome 18q. Loss of SMAD4 protein expression correlates with poor prognosis [40]. The SMAD4 protein mediates downstream TGF beta signalling events via its function as a transcription factor beta. The transforming growth factor $\beta$ (TGF- $\beta$ ) pathway acts as a double-edged sword in tumorigenesis. TGF- $\beta$ is a potent tumour suppressor by constraining epithelial cell growth but is also important in the induction of epithelial-tomesenchymal transition (EMT), thereby enhancing invasiveness and metastasis. Recent data suggest that TGF- $\beta$ signalling can be correlated with resistance against both targeted and conventional anticancer agents [41].

Deleted in colorectal cancer, gene (DCC) at 18q21.2 encodes a 170-190 $\mathrm{kDa}$ protein of the immunoglobulin superfamily and havea role in the regulation of cell adhesion and migration [42]. Genetic and epigenetic alterations collaborate in transcriptional silencing of DCC. Therefore, DDC was suggested as a candidate gene for targeted inactivation by chromosome 18q LOH [43]. 


\section{Epigenetic changes in colorectal carcinogenesis}

Different mutational defects in colorectal tumours lead to alterations in cellular signalling cascades and transcriptional regulation and consequently to many changes in gene expression in the neoplastic cells [44]. But it's clear that non-mutational or epigenetic mechanisms have also very important roles in cancer development process. By the genomic view of colorectal carcinogenesis, CRC genomes has different types of genomic alterations (oncogenic drivers?) from small-scale changes (i.e., point mutations or small index) to large-scale chromosomal copy number changes or rearrangements [45].

\section{DNA methylation}

Concordant methylation of the CG di-nucleotides in the promoter region of multiple genes is called CpG Island Methylator Phenotype (CIMP). Patients with CIMP tumours have distinct clinical and pathological characteristics.CRCs can also be classified based on the presence of MSI and CIMP [10]. Such a classification has five molecular subtypes, each with a different molecular profile and clinicopathological features. These are: 1. CIMP high/MSI high (12\% of CRC); originates in serrated adenomas and is characterized by BRAF mutation and MLH1 methylation. 2. CIMP high/MSI low or microsatellite stable (8\%); originates in serrated adenomas and is characterized by BRAF mutation and methylation of multiple genes. 3 . CIMP low/MSI low or microsatellite stable (20\%); originates in tubular, tubulovillus, or serrated adenomas and is characterized by chromosomal instability (CIN), K-ras mutation, and MGMT methylation [46]. Next group are sessile serrated adenomas (SSA) which have distinct molecular and pathological changes than traditional adenomas. SSA are progressing in cancer trough different pathway-the serrated neoplasia pathway. Fifth group are CIMP negative/microsatellite stable (57\%);originates in traditional adenoma and they are characterized by CIN $[46,47]$.

Cancer genomes seemed to be overall under methylated, but some genomic loci have focal DNA hyper methylation [48,49]. Focal hyper methylation leads to transcriptional silencing, especially at the CpG islands of gene promoters.Between the putative inactivating mechanisms of tumour suppressor genes in cancer genomes it is often preferred over the inactivation by irreversible nucleotide substitutions [50]. Cancer-associated DNA methylation seems to be more dynamic than anticipated, andDNA methylation profiling has been proposed as a toolof early CRC diagnosis using non-invasive resources (i.e., bloodor stool-based) [51-54]. A unique cancer genome-associated phenomenon in which ten to hundreds of chromosomal rearrangements occur in a "one-off" cellular event is named chromothripsis [55].

Comparison between primary and metastatic CRC genomes proved that most genomic arrangements are shared both by primary and metastatic genomes, indicating that metastasis occurs quite rapidly with few additional mutational events $[55,56]$. Although the global trend in CRC cells is hypomethylation, a number of CpG rich promoters show increased methylation with associated transcriptional silencing of the downstream gene. A large proportion of CRCs show hyper methylation and transcriptional silencing of potential tumour suppressor genes such as $\mathrm{H} 1 \mathrm{Cl}$ on chromosome $17 \mathrm{p}$ and Wnt signalling antagonists.Subset of CRCs shows coordinate hypermethylation of numerous promoters' genes suggesting that regulation of DNA methylation may be globally disrupted. Also, adenomatous precursor lesions of CIMP cancers show a distinct histologic phenotype, SSAa (sessile serrated adenomas). In SSAs B-Raf mutations are commonly observed as grow of cancers [57]. Global DNA hypo-methylation also plays an important role in CRC development, possibly through hypo methylation-induced genomic instability. A classic example of hypo methylation and CRC development is LINE-1. LINE-1 or L1 retro transposons account for about $17 \%$ of human genome; their methylation status is an important indicator of global DNA methylation level. CpG sites (located within the LINE-1 repetitive DNA elements) in association with tumour genesis tend to undergo dimethylation, [58]. LINE-1 methylation is associated with poor prognosis [58].

Epigenetic inactivation of MMR genes most commonly affects hypermethylation of the MLH1 promoter. These tumours often initially present as SSAs, show hypermethylation of numerous genes (CpG islan methylation phenotype) and B-Raf mutations. In both familial and sporadic MSI-H tumours inactivation of genes with repetitive elements (microsatellites) in their coding sequence might contribute to tumour progression (e.g., TGFbetaIIR, BAX, and HNPCC) [59].

\section{Posttranslational modifications of histones}

Among seven distinct types of histone modifications, acetylation and methylation are most extensively characterized ones that are involved in CRC pathogenesis [60-62].

Histone acetylation is reversible modifications of lysine residues on histone "tails" and is controlled by histone acetyltransferase (HATs) and histone deacetylases (HDACs) [62]. HDACs are involved in multiple signalling pathways and they are present in numerous repressive chromatin complexes; they also play key roles in CRC development $[63,64]$. Nuclear expression of HDAC2 was observed in $81.9 \%$ of CRCs, vs $53.1 \%$ of normal tissues, respectively [65]. A genomic-wide analysis found out thatmicroRNAs (miRNAs) ismethylated in human colon carcinoma cells and it is known that DNA methylation and histone acetylation often act in concert to mediate gene expression in CRC cells leading to 5-FU resistance in CRC cells [66-68]. We need more efforts to understand the various epigenetic mechanisms and their cross-talk in the context of 5-FU resistance in CRC cells, which is one of the frontlines in CRC research and drug development.

The BRAF pathway has become a target of interest for molecular therapy, and many B-Raf specific inhibitors display a cytostatic response inducing senescence and are susceptible to acquired resistance $[69,70]$. Therapy with anti-EGFR antibodies is desirable in patients with advanced CRC and absence of K-ras or B-Raf mutation. MSI status is associated with significantly better prognosis. Defining tumour phenotype MSI or MSS and testing for the presence or absence of $18 \mathrm{q}$ chromosome deletion is very much desirable in standard 5-FUbased therapy. Chemotherapy FOLFOX4 regiment did not alter survival of patients with MSI [71]. Studies on CRC biomarkers need to continue to closely examine the relationship between therapy and CRC curability. Since personalized medicine has become more clinically relevant in the last few years, genotyping of tumours is of high importance because anticancer therapy which will be used on specific targets appears to be the future of CRC therapy. 


\section{Conclusion}

CRC is a genomic disorder in which various types of genomic alterations, such as point mutations, genomic rearrangements, gene fusions, or chromosomal copy number alterations, can contribute to the initiation and progression of the disease. CRC genomes harbour various types of genomic alterations which may contribute to colorectal carcinogenesis as oncogenic drivers, but the full spectrum of driver genomic alterations in CRC genomes is still incomplete. Several studies have suggested that $\mathrm{LOH}$ of specific chromosomes, such as chromosome $8 \mathrm{q}, 17 \mathrm{p}$ or $18 \mathrm{q}$ may be useful in assessing prognosis in patients with stage II and III colorectal cancer.Studies on CRC biomarkers need to continue to closely examine the relationship between therapy and CRC curability. Nowadays combination therapy is likely to be the most effective management plan for the treatment of B-raf-mutated tumours. Defining tumour phenotype MSI or MSS and testing for the presence or absence of $18 \mathrm{q}$ chromosome deletion is very much desirable in standard 5-FU-based therapy. Future of CRC therapy is connected to tumour genotyping because anticancer therapy will be used on specific targets.

\section{References}

1. Olivier M, Hollstein M, Hainaut P (2010) TP53 mutations in human cancers: origins, consequences, and clinical use. Cold Spring Harb Perspect Biol 2: a001008.

2. Kopetz S, Chang GJ, Overman MJ, Eng C, Sargent DJ, et al. (2009) Improved survival in metastatic colorectal cancer is associated with adoption of hepatic resection and improved chemotherapy. J Clin Oncol 27: 3677-3683.

3. Lutz W, Nowakowska-Swirta E (2002) Gene p53 mutations, protein p53, and anti-p53 antibodies as biomarkers of cancer process. Int J Occup Med Environ Health 15: 209-218.

4. Petitjean A, Mathe E, Kato S, Ishioka C, Tavtigian SV, et al. (2007) Impact of mutant p53 functional properties on TP53 mutation patterns and tumor phenotype: lessons from recent developments in the IARC TP53 database. Hum Mutat 28: 622-629.

5. Béroud C, Collod-Béroud G, Boileau C, Soussi T, Junien C (2000) UMD (Universal mutation database): a generic software to build and analyze locus-specific databases. Hum Mutat 15: 86-94.

6. Soussi T, Dehouche K, Béroud C (2000) p53 website and analysis of p53 gene mutations in human cancer: forging a link between epidemiology and carcinogenesis. Hum Mutat 15: 105-113.

7. Luo Y, Wang L, Wang J (2013) Developing proteomics-based biomarkers for colorectal neoplasms for clinical practice: opportunities and challenges. Proteomics Clin Appl 7: 30-41.

8. de Wit M, Fijneman RJ, Verheul HM, Meijer GA, Jimenez CR (2013) Proteomics in colorectal cancer translational research: biomarker discovery for clinical applications. Clin Biochem 46: 466-479.

9. Al-Sohaily S, Biankin A, Leong R, Kohonen-Corish M, Warusavitarne J (2012) Molecular pathways in colorectal cancer. J Gastroenterol Hepatol 27: $1423-1431$.

10. Jass JR (2007) Classification of colorectal cancer based on correlation of clinical, morphological and molecular features. Histopathology 50: 113-130.

11. Sheffer M, Bacolod MD, Zuk O, Giardina SF, Pincas H, et al. (2009) Association of survival and disease progression with chromosomal instability: a genomic exploration of colorectal cancer. Proc Natl Acad Sci U S A 106: 7131-7136.

12. Thiagalingam S, Laken S, Willson JK, Markowitz SD, Kinzler KW, et al. (2001) Mechanisms underlying losses of heterozygosity in human colorectal cancers. Proc Natl Acad Sci U S A 98: 2698-2702.

13. Wildrick DM (1989) Molecular genetic studies of colon cancer. Hematol Oncol Clin North Am 3: 1-18.
14. Pino MS, Chung DC (2010) The chromosomal instability pathway in colon cancer. Gastroenterology 138: 2059-2072.

15. Mouradov D, Domingo E, Gibbs P, Jorissen RN, Li S, et al. (2013) Survival in stage II/III colorectal cancer is independently predicted by chromosomal and microsatellite instability, but not by specific driver mutations. Am J Gastroenterol 108:1785-1793.

16. Aaltonen LA, Peltomäki P, Leach FS, Sistonen P, Pylkkänen L, et al. (1993) Clues to the pathogenesis of familial colorectal cancer. Science 260: 812-816.

17. Ionov Y, Peinado MA, Malkhosyan S, Shibata D, Perucho M (1993) Ubiquitous somatic mutations in simple repeated sequences reveal a new mechanism for colonic carcinogenesis. Nature 363: 558-561.

18. Boland CR, Goel A (2010) Microsatellite instability in colorectal cancer. Gastroenterology 138: 2073-2087.

19. Kohonen-Corish MR1, Daniel JJ, Chan C, Lin BP, Kwun SY, et al. (2005) Low microsatellite instability is associated with poor prognosis in stage C colon cancer. J Clin Oncol 23: 2318-2324.

20. Iacopetta B, Grieu F, Amanuel B (2010) Microsatellite instability in colorectal cancer. Asia Pac J Clin Oncol 6: 260-269.

21. Kumar K, Brim H, Giardiello F, Smoot DT, Nouraie M, et al. (2009) Distinct BRAF (V600E) and KRAS mutations in high microsatellite instability sporadic colorectal cancer in African Americans. Clin Cancer Res 15: 1155-1161.

22. Buecher B, Cacheux W, Rouleau E, Dieumegard B, Mitry E, et al. (2013) Role of microsatellite instability in the management of colorectal cancers. Dig Liver Dis 45: 441-449.

23. Wang JY, Wang YH, Jao SW, Lu CY, Kuo CH, et al. (2006) Molecular mechanisms underlying the tumorigenesis of colorectal adenomas: correlation to activated K-ras oncogene. Oncol Rep 16: 1245-1252.

24. Pajkos G, Kiss I, Sándor J, Ember I, Kisházi P (2000) The prognostic value of the presence of mutations at the codons 12, 13, 61 of K-ras oncogene in colorectal cancer. Anticancer Res 20: 1695-1701.

25. Brink M, de Goeij AF, Weijenberg MP, Roemen GM, Lentjes MH, et al. (2003) K-ras oncogene mutations in sporadic colorectal cancer in The Netherlands Cohort Study. Carcinogenesis 24: 703-710.

26. Mao X, Young BD, Lu YJ (2007) The application of single nucleotide polymorphism microarrays in cancer research. Curr Genomics 8: 219-228.

27. Rosty C, Young JP, Walsh MD, Clendenning M, Walters RJ, et al. (2013) Colorectal carcinomas with KRAS mutation are associated with distinctive morphological and molecular features. Mod Pathol 26: 825-834.

28. Vogelstein B, Fearon ER, Hamilton SR, Kern SE, Preisinger AC, et al. (1988) Genetic alterations during colorectal-tumor development. N Engl J Med 319: 525-532.

29. Grady WM, Markowitz SD (2002) Genetic and epigenetic alterations in colon cancer. Annu Rev Genomics Hum Genet 3: 101-128.

30. Green DR, Kroemer G (2009) Cytoplasmic functions of the tumour suppressor p53. Nature 458: 1127-1130.

31. Fearon ER, Vogelstein B (1990) A genetic model for colorectal tumorigenesis. Cell 61: 759-767.

32. Powell SM, Zilz N, Beazer-Barclay Y, Bryan TM, Hamilton SR, et al. (1992) APC mutations occur early during colorectal tumorigenesis. Nature 359: 235-237.

33. Kolligs FT, Bommer G, Göke B (2002) Wnt/beta-catenin/tcf signaling: a critical pathway in gastrointestinal tumorigenesis. Digestion 66: 131-144.

34. Segditsas S, Tomlinson I (2006) Colorectal cancer and genetic alterations in the Wnt pathway. Oncogene 25: 7531-7537.

35. Fukuyama R, Niculaita R, Ng KP, Obusez E, Sanchez J, et al. (2008) Mutated in colorectal cancer, a putative tumor suppressor for serrated colorectal cancer, selectively represses beta-catenin-dependent transcription. Oncogene 27: 6044-6055.

36. Liu J, Liao Y, Ma K, Wang Y, Zhang G, et al. (2013) PI3K is required for the physical interaction and functional inhibition of NF- $\hat{I}^{\circ} B$ by $\hat{\mathrm{I}}^{2}$-catenin in colorectal cancer cells. Biochem Biophys Res Commun 434: 760-766. 
37. Howarth K, Ranta S, Winter E, Teixeira A, Schaschl H, et al. (2009) A mitotic recombination map proximal to the APC locus on chromosome 5q and assessment of influences on colorectal cancer risk. BMC Med Genet 10: 54 .

38. Lanza G, Matteuzzi M, Gafá R, Orvieto E, Maestri I, et al. (1998) Chromosome 18q allelic loss and prognosis in stage II and III colon cancer. Int J Cancer 79: 390-395.

39. Wang W, Wang GQ, Sun XW, Chen G, Li YF, et al. (2010) Prognostic values of chromosome $18 \mathrm{q}$ microsatellite alterations in stage II colonic carcinoma. World J Gastroenterol 16: 6026-6034.

40. McDermott U, Longley DB, Johnston PG (2002) Molecular and biochemical markers in colorectal cancer. Ann Oncol 13 Suppl 4: 235-245.

41. Brunen D, Willems SM, Kellner U, Midgley R, Simon I, et al. (2013) TGF- $\hat{I}^{2}$ : an emerging player in drug resistance. Cell Cycle 12: 2960-2968.

42. Martín M, Simon-Assmann P, Kedinger M, Martin M, Mangeat P, et al. (2006) DCC regulates cell adhesion in human colon cancer derived HT-29 cells and associates with ezrin. Eur J Cell Biol 85: 769-783.

43. Derks S, Bosch LJ, Niessen HE, Moerkerk PT, van den Bosch SM, et al. (2009) Promoter CpG island hypermethylation- and H3K9me3 and H3K27me3-mediated epigenetic silencing targets the deleted in colon cancer (DCC) gene in colorectal carcinogenesis without affecting neighboring genes on chromosomal region 18q21. Carcinogenesis 30:1041-1048

44. Crea F, Nobili S, Paolicchi E, Perrone G, Napoli C, et al. (2011) Epigenetics and chemoresistance in colorectal cancer: an opportunity for treatment tailoring and novel therapeutic strategies. Drug Resist Updat 14: $280-296$

45. Migheli F, Migliore L (2012) Epigenetics of colorectal cancer. Clin Genet 81: 312-318.

46. Jemal A1, Siegel R, Xu J, Ward E (2010) Cancer statistics, 2010. CA Cancer J Clin 60: 277-300.

47. Leedham S, East JE, Chetty R (2013) Diagnosis of sessile serrated polyps/ adenomas: what does this mean for the pathologist, gastroenterologist and patient? J Clin Pathol 66: 265-268.

48. Herman JG, Baylin SB (2003) Gene silencing in cancer in association with promoter hypermethylation. N Engl J Med 349: 2042-2054.

49. Jones PA, Baylin SB (2002) The fundamental role of epigenetic events in cancer. Nat Rev Genet 3: 415-428.

50. Chan TA, Glockner S, Yi JM, Chen W, Van Neste L, et al. (2008) Convergence of mutation and epigenetic alterations identifies common genes in cancer that predict for poor prognosis. PLoS Med 5: el14.

51. Frigola J, Song J, Stirzaker C, Hinshelwood RA, Peinado MA, et al. (2006) Epigenetic remodeling in colorectal cancer results in coordinate gene suppression across an entire chromosome band. Nat Genet 38: 540-549.

52. Irizarry RA, Ladd-Acosta C, Wen B, Wu Z, Montano C, et al. (2009) The human colon cancer methylome shows similar hypo- and hypermethylation at conserved tissue-specific CpG island shores. Nat Genet 41: 178-186.

53. Glöckner SC, Dhir M, Yi JM, McGarvey KE, Van Neste L, et al. (2009) Methylation of TFPI2 in stool DNA: a potential novel biomarker for the detection of colorectal cancer. Cancer Res 69: 4691-4699.

54. Warren JD, Xiong W, Bunker AM, Vaughn CP, Furtado LV, et al. (2011) Septin 9 methylated DNA is a sensitive and specific blood test for colorectal cancer. BMC Med 9: 133.
55. Stephens PJ, Greenman CD, Fu B, Yang F, Bignell GR, et al. (2011) Massive genomic rearrangement acquired in a single catastrophic event during cancer development. Cell 144: 27-40.

56. Jones S, Chen WD, Parmigiani G, Diehl F, Beerenwinkel N, et al. (2008) Comparative lesion sequencing provides insights into tumor evolution. Proc Natl Acad Sci U S A 105: 4283-4288.

57. Burnett-Hartman AN, Newcomb PA, Potter JD, Passarelli MN, Phipps AI, et al. (2013) Genomic aberrations occurring in subsets of serrated colorectal lesions but not conventional adenomas. Cancer Res 73: 2863-2872.

58. Estécio MR, Gharibyan V, Shen L, Ibrahim AE, Doshi K, et al. (2007) LINE-1 hypomethylation in cancer is highly variable and inversely correlated with microsatellite instability. PLoS One 2: e399.

59. Grady WM (2004) Genomic instability and colon cancer. Cancer Metastasis Rev 23: 11-27.

60. Nakazawa T, Kondo T, Ma D, Niu D, Mochizuki K, et al. (2012) Global histone modification of histone $\mathrm{H} 3$ in colorectal cancer and its precursor lesions. Hum Pathol 43: 834-842.

61. Li Q, Chen H (2012) Silencing of Wnt5a during colon cancer metastasis involves histone modifications. Epigenetics 7: 551-558.

62. Binder H, Steiner L, Przybilla J, Rohlf T, Prohaska S, et al. (2013) Transcriptional regulation by histone modifications: towards a theory of chromatin re-organization during stem cell differentiation. Phys Biol 10: 026006.

63. Weichert W, Roske A, Niesporek S, Noske A, Buckendahl AC, et al. (2008) Class I histone deacetylase expression has independent prognostic impact in human colorectal cancer: Specific role of class I histone deacetylases in vitro and in vivo. Clin. Cancer Res 14:1669-1677.

64. Ishihama K, Yamakawa M, Semba S, Takeda H, Kawata S, et al. (2007) Expression of $\mathrm{HDAC} 1$ and $\mathrm{CBP} / \mathrm{p} 300$ in human colorectal carcinomas. J Clin Pathol 60: 1205-1210.

65. Nosho K, Shima K, Irahara N, Kure S, Firestein R, et al. (2009) SIRT1 histone deacetylase expression is associated with microsatellite instability and $\mathrm{CpG}$ island methylator phenotype in colorectal cancer. Mod Pathol 22: 922-932.

66. Crea F, Nobili S, Paolicchi E, Perrone G, Napoli C, et al. (2011) Epigenetics and chemoresistance in colorectal cancer: an opportunity for treatment tailoring and novel therapeutic strategies. Drug Resist Updat 14: $280-296$.

67. Yan H, Choi AJ, Lee BH, Ting AH (2011) Identification and functional analysis of epigenetically silenced microRNAs in colorectal cancer cells. PLoS One 6: e20628.

68. Yang D, Torres CM, Bardhan K, Zimmerman M, McGaha TL, et al. (2012) Decitabine and vorinostat cooperate to sensitize colon carcinoma cells to Fas ligand-induced apoptosis in vitro and tumor suppression in vivo. J Immunol 188: 4441-4449.

69. Wellbrock C, Hurlstone A (2010) BRAF as therapeutic target in melanoma. Biochem Pharmacol 80: 561-567.

70. Hatzivassiliou G, Song K, Yen I, Brandhuber BJ, Anderson DJ, et al. (2010) RAF inhibitors prime wild-type RAF to activate the MAPK pathway and enhance growth. Nature 464: 431-435.

71. Des Guetz G, Lecaille C, Mariani P, Bennamoun M, Uzzan B, et al. (2010) Prognostic impact of microsatellite instability in colorectal cancer patients treated with adjuvant FOLFOX. Anticancer Res 30: 4297-4301. 